Proceedings of the International School and Conference on Optics and Optical Materials, ISCOM07, Belgrade, Serbia, September 3-7, 2007

\title{
Beam Propagation in Nematic Liquid Crystals
}

\author{
A.I. StriniC ${ }^{a *}$ AND M.R. Belic ${ }^{b}$ \\ ${ }^{a}$ Institute of Physics, Pregrevica 118, 11080 Belgrade, Serbia \\ ${ }^{b}$ Texas A\&M University at Qatar, P.O. Box 23874, Doha, Qatar
}

We investigate the behavior of beams propagating in nematic liquid crystals. A spatiotemporal model for the beam propagation and the director reorientation in a nematic liquid crystal is treated numerically in three spatial dimensions and time. We demonstrate the formation of stable solitons in a narrow threshold region of beam intensities for a set of fixed parameters and display soliton breathing. Below the threshold region the beams diffract, above the region spatiotemporal instabilities are observed, as the input intensity and the optical and static permittivity anisotropies of the liquid crystal molecules are increased. We demonstrate the filamentation of solitons above the threshold with increasing input intensity.

PACS numbers: 42.65.Tg, 42.65.Sf, 42.70.Df

\section{Introduction}

Nematic liquid crystals (NLC) are ubiquitous materials, found in many consumer electronic devices. A nematic liquid crystal possesses some properties of both liquids and solids. It contains rod-like molecules which exhibit orientational alignment without positional order. They behave in a fluid-like fashion, but display long-range order that is characteristic of crystals [1].

A unique property of NLC is their ability to change optical characteristics under the action of an external electric field, which enables the macroscopic reorientation of the director tilt angle $\theta$ in NLC. In other words, the light incident on an NLC modifies the electric permittivity tensor, leading to the reorientational nonlinearity.

NLC are known to exhibit enormous optical nonlinearities, owing to their large refractive index anisotropy, coupled with the optically-induced collective molecular reorientation. These qualities make them suitable for investigation using lasers of relatively low power and low-cost detection equipment. For that reason

*corresponding author; e-mail: strinic@phy.bg.ac.yu 
the propagation of self-focused beams [2] in NLC have been the subject of considerable interest in recent years, from both experimental $[3,4]$ and theoretical $[5-8]$ points of view.

We consider here single beam propagation in NLC. We investigate beam propagation for different values of control parameters (such as the input intensity, the optical and static permittivity anisotropies of the liquid-crystal molecules and the input beam width). Stable beams are observed in a very narrow threshold region of control parameters. We display soliton breathing - the beam preserves its new shape but its characteristic width and maximum intensity breathe sinusoidally as it propagates. By increasing the input beam intensity, spatiotemporal instabilities and chaotic behavior are observed.

\section{The model}

The distortion of molecular orientation in NLC can be interpreted by a reorientation angle $\theta$ of the director in the transverse plane. In the presence of an externally applied (low frequency) voltage the spatial evolution of a slowly-varying beam envelope $A$, linearly polarized along the $x$ axis and propagating along the $z$ axis, is well described by the paraxial wave equation [9]:

$$
2 \mathrm{i} k \frac{\partial A}{\partial \mathrm{z}}+\Delta_{x, y} A+k_{0}^{2} \Delta \varepsilon^{\mathrm{OPT}}\left(\sin ^{2} \theta-\sin ^{2} \theta_{0}\right) A=0,
$$

where $k=k_{0} n_{0}$ is the wave vector in the medium, $\Delta_{x, y}$ is the transverse Laplacian, and $\Delta \varepsilon^{\mathrm{OPT}}=n_{\mathrm{e}}^{2}-n_{0}^{2}$ is the optical permittivity anisotropy of the liquid-crystal molecules. The temporal evolution of the angle of reorientation $\theta$ is given by the diffusion equation $[4,10]$ :

$$
\gamma \frac{\partial \theta}{\partial t}=K \Delta_{x, y} \theta+\frac{\varepsilon_{0}}{2}\left(\Delta \varepsilon^{\mathrm{DC}}\left|E^{\mathrm{DC}}\right|^{2}+\Delta \varepsilon^{\mathrm{OPT}} \frac{|A|^{2}}{2}\right) \sin (2 \theta)
$$

with the boundary condition

$$
\theta(x=-D / 2)=\theta(x=D / 2)=2^{\circ} .
$$

Here $\gamma$ is the viscous coefficient and $\mathrm{K}$ is Frank's elastic constant. $E^{\mathrm{DC}}=V / D$ is the applied field strength, $D$ is the cell thickness, $V$ is the applied bias voltage, $\Delta \varepsilon^{\mathrm{DC}}$ is the static permittivity anisotropy of the liquid-crystal molecules. $\theta$ is the overall tilt angle (the total orientation of the molecules with respect to the $z$ axis), owing to both light and voltage: $\theta=\theta_{0}+\hat{\theta}$, where the angle $\theta_{0}$ accounts for the molecular orientation induced by the static electric field only, while the quantity $\hat{\theta}$ corresponds to the optically induced molecular reorientation. Hard boundary conditions on the molecular orientation at the transverse borders of the liquid-crystal cell are introduced by the relation (3).

Using the rescaling $\mathrm{z}=z k \mathrm{x}_{0}^{2}, \mathrm{x}=x \mathrm{x}_{0}, \mathrm{y}=y \mathrm{x}_{0}$ and $\mathrm{t}=T \tau$, we transform the equations into a dimensionless form

$$
2 \mathrm{i} k \frac{\partial A}{\partial z}+\Delta_{x, y} A+k_{0}^{2} x_{0}^{2} \Delta \varepsilon^{\mathrm{OPT}}\left(\sin ^{2} \theta-\sin ^{2} \theta_{0}\right) A=0
$$




$$
\frac{2 \gamma x_{0}^{2}}{K \tau} \frac{\partial \theta}{\partial T}=2 \Delta_{x, y} \theta+\left(\frac{\varepsilon_{0} x_{0}^{2} \Delta \varepsilon^{\mathrm{DC}}\left|E^{\mathrm{DC}}\right|^{2}}{K}+k_{0}^{2} x_{0}^{2} \Delta \varepsilon^{\mathrm{OPT}} \frac{\varepsilon_{0}|A|^{2}}{2 K k_{0}^{2}}\right) \sin (2 \theta),
$$

where $\tau$ is the director relaxation time and $x_{0}$ the transverse scaling length. Using abbreviations

$$
\alpha=k_{0}^{2} x_{0}^{2} \Delta \varepsilon^{\mathrm{OPT}}, \quad \beta=\frac{\varepsilon_{0} x_{0}^{2} \Delta \varepsilon^{\mathrm{DC}}\left|E^{\mathrm{DC}}\right|^{2}}{K} \quad \text { and } \quad|A|^{2}=\frac{\varepsilon_{0}|A|^{2}}{2 K k_{0}^{2}},
$$

we obtain Eqs. (7) and (8) which form the basis of our model

$$
\begin{aligned}
& 2 \mathrm{i} \frac{\partial A}{\partial z}+\Delta_{x, y} A+\alpha\left(\sin ^{2} \theta-\sin ^{2} \theta_{0}\right) A=0, \\
& \frac{2 \gamma x_{0}^{2}}{K \tau} \frac{\partial \theta}{\partial T}=2 \Delta_{x, y} \theta+\left(\beta+\alpha|A|^{2}\right) \sin (2 \theta) .
\end{aligned}
$$

By solving these equations we will describe the beam propagation in both space and time. We develop a novel numerical procedure, based on fast Fourier transform (FFT), utilizing our prior experience in treating the beam propagation in nematic liquid crystals [5]. The novelty is that we treat concurrently the system of coupled partial differential equations in both space and time. Our procedure is uniquely suited for observing the dynamical states in slowly-varying physical systems, where the fast optical fields are slaved to the slow change in the nonlinearity.

In all simulations the following data are kept fixed: the diffraction length $L_{d}=k x_{0}^{2}=79 \mu \mathrm{m}$, the propagation distance $L=20 L_{d}=1.5 \mathrm{~mm}$, the transverse scaling length $x_{0}=2 \mu \mathrm{m}$, the laser wavelength $\lambda=514 \mathrm{~nm}$, the elastic constant $K=12 \times 10^{-12} \mathrm{~N}$, the viscous coefficient $\gamma=0.08 \mathrm{~kg} /(\mathrm{m} \mathrm{s})$, the ordinary refractive index $n_{0}=1.53$, the cell thickness $D=75 \mu \mathrm{m}$, and the bias voltage $V=1 \mathrm{~V}$. All of these data are consistent with the values reported in experimental investigations $[4,10]$.

The initial beam widths FWHM are varied between $2.5 \mu \mathrm{m}$ and $4 \mu \mathrm{m}$. The values for the optical and static permittivity anisotropies of the liquid-crystal molecules are varied between 0.3 and 0.5 , and between 10 and 14.5 , respectively. The intensity is varied between $I=0.5 \times 10^{10} \mathrm{~V}^{2} / \mathrm{m}^{2}$ and $I=5 \times 10^{12} \mathrm{~V}^{2} / \mathrm{m}^{2}$.

The main difference between the model used in this paper and the model used previously $[5,11]$ is that now the temporal evolution of the angle of reorientation, owing to both light and voltage, is given by Eq. (2), where $\theta=\theta_{0}+\hat{\theta}$. $\theta_{0}$ is now determined in the beginning, using boundary conditions and the program SOR (successive over relaxation algorithm for solving partial differential equation $(\mathrm{PDE})$ ). Earlier it was given by an approximate formula. This provides for a more realistic physical modeling of the system.

\section{Results of numerical simulation}

Nonlocality exerts great impact on the beam propagation in NLC [12-14]. Spatiotemporal nonlocality means that the response of the medium at a particular point and in a given moment, is not determined exclusively by the wave intensity at that point and in that moment, but also depends on the wave intensity in its 
vicinity and at earlier moments. Nonlocality also exerts great influence on the modulational instability (MI).

MI represents an exponential growth of a weak spatial perturbation of the wave as it propagates. The gain causes the amplification of side bands, which leads to the breakup of the wave and the appearance of transverse localized structures through the process of filamentation. Saturable nonlocal nonlinearity tends to suppress the exponential growth of instability, decreasing the growth rate and the width of the instability band. But, it cannot eliminate the instability completely. The first experimental observation and study of MI in NLC is given in $[13,14]$.

We consider the behavior of propagating Gaussian beams in NLC. We increase the beam intensity until spatial solitons - stable beams propagating without change in the transverse profile - are located. The effect of the input intensity variation on the single Gaussian beam propagation is presented in Fig. 1.

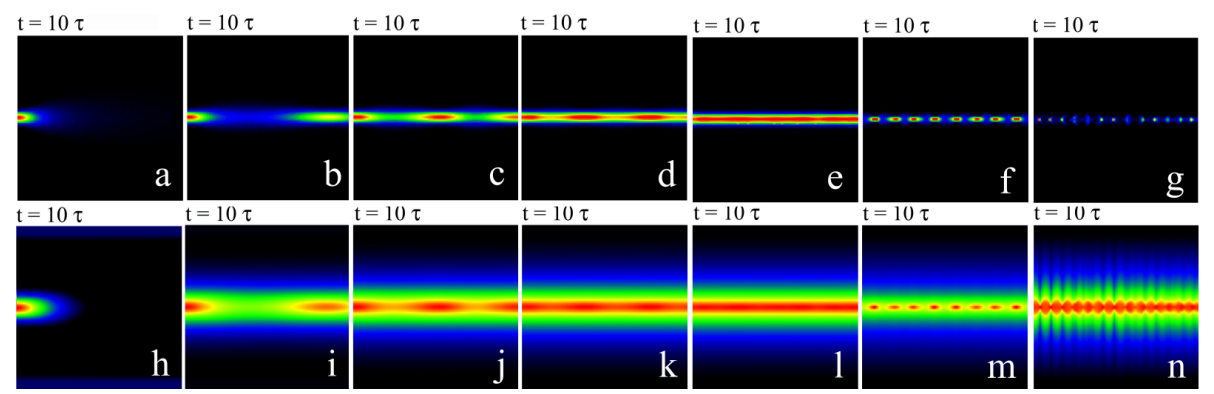

Fig. 1. Beam propagation, shown for the beams in the $(y, z)$ plane, for different input intensities. The first row shows $I(y, z)$, the second row $\hat{\theta}(y, z)$. Parameters: (a) and (h) $I=1 \times 10^{10} \mathrm{~V}^{2} / \mathrm{m}^{2}$, (b) and (i) $I=2 \times 10^{10} \mathrm{~V}^{2} / \mathrm{m}^{2}$, (c) and (j) $I=2.8 \times 10^{10} \mathrm{~V}^{2} / \mathrm{m}^{2}$, (d) and (k) $I=3.5 \times 10^{10} \mathrm{~V}^{2} / \mathrm{m}^{2}$, (e) and (l) $I=4 \times 10^{10} \mathrm{~V}^{2} / \mathrm{m}^{2}$, (f) and (m) $I=$ $1 \times 10^{11} \mathrm{~V}^{2} / \mathrm{m}^{2}$, and $(\mathrm{g})$ and $(\mathrm{n}) I=5 \times 10^{12} \mathrm{~V}^{2} / \mathrm{m}^{2}$. For all simulations $\Delta \varepsilon^{\mathrm{OPT}}=0.5$, $\Delta \varepsilon^{\mathrm{DC}}=14.5$, the initial beam widths $\mathrm{FWHM}=4 \mu \mathrm{m}$ and $L=1.5 \mathrm{~mm}$.

For smaller intensities (Fig. 1a and b) self-focusing is too weak to keep the beam tightly focused, so that it cannot pass through unchanged=4. By increasin the beam intensity (Fig. 1d and e) we achieve breathing and stable solitonic propagation, i.e. the beam preserves its new shape, but its characteristic width and maximum intensity breathe sinusoidally as it propagates. For still higher intensities we observe irregular behavior of the beam, so that it is not passing through unchanged, as a soliton. Slight width modulations, present at the onset of soliton formation, become more pronounced as the intensities are increased. In that range of intensities we see periodical spot size variation during propagation - the breathing of a spatial soliton. The width modulation pitch increases with the intensity increase (Fig. 1d-f). Eventually, the soliton is completely broken (Fig. 1f, g) by the joint action of the longitudinal modulation instability and the transversal instability, mixing in at intensities above $5 \times 10^{11} \mathrm{~V}^{2} / \mathrm{m}^{2}$ (Fig. $1 \mathrm{~g}$ ). 
Staying with the same figure, a comparison between the beam and the angle reorientation distributions shows that they change in unison. The angle reorientation distribution is always wider. This comes because of the nonlocal nature of nonlinearity. The profiles are made in the $(y, z)$ plane, at the same moment. They exhibit similar behavior to the one reported in $[15,16]$.

For the intensities represented in Fig 1, we show in Fig. 2 the intensities and $\hat{\theta}$ in the middle of the crystal, $I(0,0, z)$ and $\theta(0,0, z)$ as functions of the propagation distance. Figure 2 represents typical behavior during the propagation of optical Gaussian beams in NLC. As we can see, the soliton breathing (the maximum intensity breathes sinusoidally as the soliton propagates) is the only way of soliton propagation.
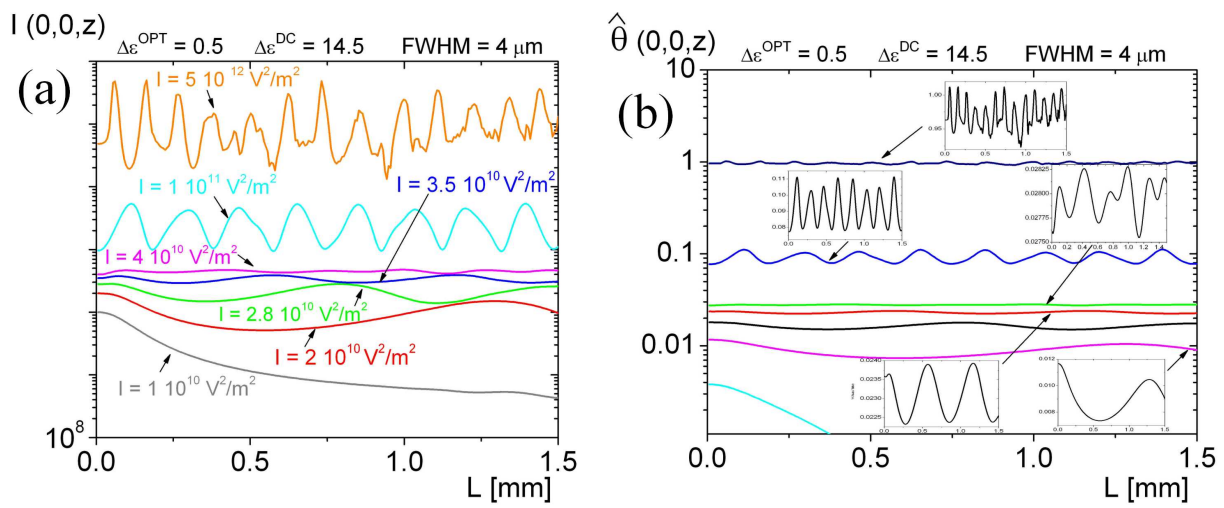

Fig. 2. Beam propagation in the $z$-direction for the intensities in the middle of the crystal, $I(0,0, z)$, and for $\hat{\theta}(0,0, z)$, shown for different input intensities from Fig. 1. Parameters: $\Delta \varepsilon^{\mathrm{OPT}}=0.5, \Delta \varepsilon^{\mathrm{DC}}=14.5$, FWHM $=4 \mu \mathrm{m}$ and $L=1.5 \mathrm{~mm}$. Insets depictvariation on the linear scale.

When the same sequence of increasing input intensity simulations is repeated for different input width FWHM, for fixed parameters $\Delta \varepsilon^{\mathrm{OPT}}=0.4$ and $\Delta \varepsilon^{\mathrm{DC}}=$ 14.5, we find similar behavior. For each input beam width the appropriate input beam intensity can be found for the existence of breathing soliton. In Fig. 3a we show the cases for the breathing soliton propagation, for FWHM $=2.5 \mu \mathrm{m}, 3 \mu \mathrm{m}$, $3.5 \mu \mathrm{m}$, and $4 \mu \mathrm{m}$.

If the same sequence of increasing input intensity simulations is repeated for smaller birefringence, $\Delta \varepsilon^{\mathrm{OPT}}=0.4$ and $\Delta \varepsilon^{\mathrm{OPT}}=0.3\left(\Delta \varepsilon^{\mathrm{DC}}=14.5\right)$, and for thefixed input beam width $\mathrm{FWHM}=4 \mu \mathrm{m}$, a similar behavioral pattern is observed (Fig. 3b). However, the appropriate input beam intensity for the emergence of breathing soliton appears at a higher intensity. For smaller $\Delta \varepsilon^{\mathrm{DC}}=10$ we see (Fig. 3b) an increase in the intensity which is needed for the existence of the breathing soliton stable propagation. 

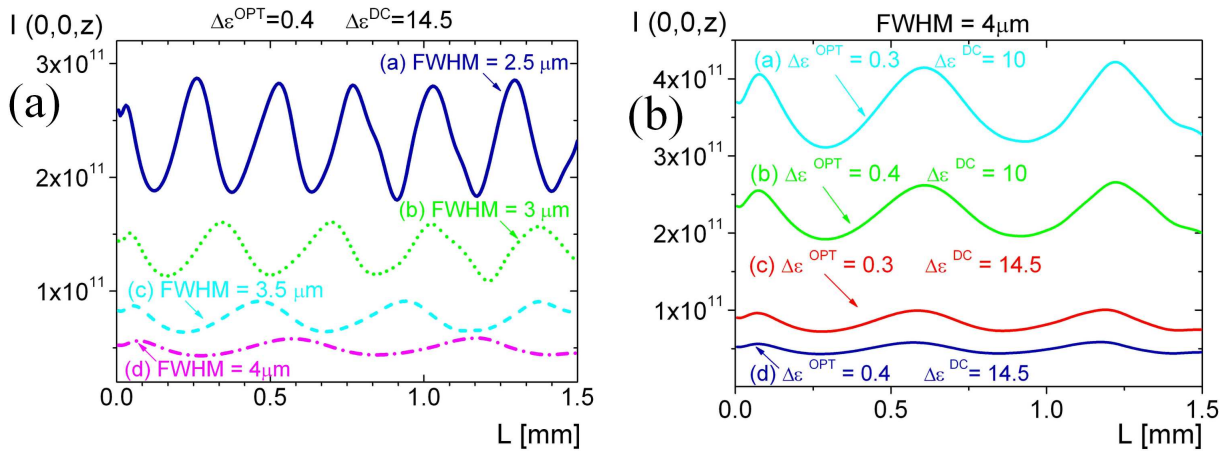

Fig. 3. (a) Soliton propagation in the $(0,0, z)$ direction: the input beam intensity profile is shown for different input widths FWHM. For each input beam width appropriate input beam intensity is found for the existence of a breathing soliton. Parameters: $\Delta \varepsilon^{\mathrm{OPT}}=0.4, \Delta \varepsilon^{\mathrm{DC}}=14.5$, and $L=1.5 \mathrm{~mm}$. (b) Soliton propagation in the $(0,0, z)$ direction, for different $\Delta \varepsilon^{\mathrm{OPT}}$ and $\Delta \varepsilon^{\mathrm{DC}}$. Four cases are shown: (a) $\Delta \varepsilon^{\mathrm{OPT}}=0.3$ and $\Delta \varepsilon^{\mathrm{DC}}=10$, (b) $\Delta \varepsilon^{\mathrm{OPT}}=0.4$ and $\Delta \varepsilon^{\mathrm{DC}}=10$, (c) $\Delta \varepsilon^{\mathrm{OPT}}=0.3$ and $\Delta \varepsilon^{\mathrm{DC}}=14.5$, and (d) $\Delta \varepsilon^{\mathrm{OPT}}=0.4$ and $\Delta \varepsilon^{\mathrm{DC}}=14.5$. Other parameters: $\mathrm{FWHM}=4 \mu \mathrm{m}$ and $L=1.5 \mathrm{~mm}$.
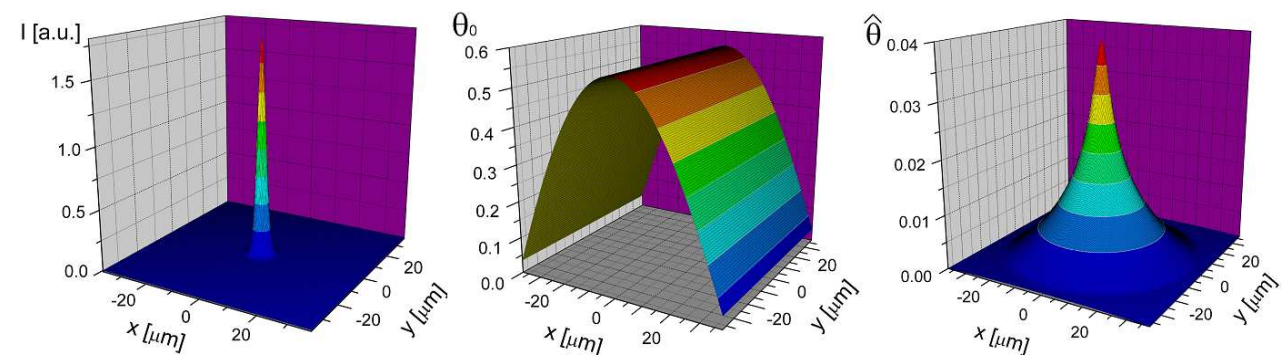

Fig. 4. Breathing soliton transverse profiles at the exit $(x, y)$ plane, (a) the optical field intensity $I(x, y)$ in in arbitrary units; (b) the molecular orientation induced by the electric field only $\theta_{0}(x, y)$ and (c) the optically induced molecular reorientation $\hat{\theta}(x, y)$. The input intensity $I=8.6 \times 10^{10} \mathrm{~V}^{2} / \mathrm{m}^{2}, \mathrm{FWHM}=3.5 \mu \mathrm{m}, L=1.5 \mathrm{~mm}$, $\Delta \varepsilon^{\mathrm{OPT}}=0.4$, and $\Delta \varepsilon^{\mathrm{DC}}=14.5$.

For the case $\varepsilon_{a}=0.4, \Delta \varepsilon^{\mathrm{DC}}=14.5$, and FWHM $=3.5 \mu \mathrm{m}$ we show in Fig. 4 the breathing soliton transverse profiles of the optical field intensity, the molecular orientation induced by the electric field $\theta_{0}$, and the optically induced molecular reorientation $\hat{\theta}$. We can notice a perfect radial symmetry of $\hat{\theta}$ and $I$ profiles in the soliton regime. Finally, we considered the propagation in NLC in the steady state, and also found similar behavior — the existence of breathing soliton as a stable propagation mode of beams in NLC. 


\section{Conclusion}

We report on the behavior of beam propagation in NLC, for different values of beam parameters. We demonstrate the formation of stable solitons in a narrow threshold region of beam intensities for fixed parameters and display soliton breathing. We observe modulational instabilities and strong beam filamentation. We change the input beam width FWHM for fixed parameters and for the fixed FWHM we change the optical and static permittivity anisotropies of liquid-crystal molecules, and for each of those cases we find appropriate input beam intensities for the existence of breathing solitons.

\section{Acknowledgments}

The authors acknowledge a financial support of the Ministry of Science of the Republic of Serbia (grant No. OI 141031).

\section{References}

[1] I.C. Khoo, Liquid Crystals: Physical Properties and Nonlinear Optical Phenomena, Wiley, New York 1995.

[2] Y.S. Kivshar, G.P. Agrawal, Optical Solitons, Academic Press, San Diego 2003.

[3] M. Peccianti, C. Conti, G. Assanto, A. De Luca, C. Umeton, J. Nonlin. Opt. Phys. Mater. 12, 525 (2003).

[4] X. Hutsebaut, C. Cambournac, M. Haelterman, J. Beeckman, K. Neyts, J. Opt. Soc. Am. B 22, 1424 (2005).

[5] A.I. Strinić, D.V. Timotijević, D. Arsenović, M.S. Petrović, M.R. Belić, Opt. Express 13, 493 (2005).

[6] P.D. Rasmussen, O. Bang, W. Krolikowski, Phys. Rev. E 72, 066611 (2005).

[7] G. D'Alessandro, A.A. Wheeler, Phys. Rev. A 67, 023816 (2003).

[8] J.F. Henninot, M. Debailleul, M. Warenghem, Mol. Cryst. Liq. Cryst. 375, 631 (2002).

[9] M. Peccianti, A. De Rossi, G. Assanto, A. De Luca, C. Umeton, I. Khoo, Appl. Phys. Lett. 77, 7 (2000).

[10] J. Beeckman, K. Neyts, X. Hutsebaut, C. Cambournac, M. Haelterman, Opt. Express 12, 1011 (2004).

[11] A. Strinić, D. Jović, M. Petrović, D. Timotijević, N. Aleksić, M. Belić, Opt. Express 14, 12310 (2006).

[12] W. Krolikowski, O. Bang, N. Nikolov, D. Neshev, J. Wyller, J. Rasmussen, D. Edmundson, J. Opt. B 6, S288 (2004).

[13] C. Conti, M. Peccianti, G. Assanto, Phys. Rev. Lett. 91, 073901 (2003).

[14] M. Peccianti, C. Conti, G. Assanto, Phys. Rev. E 68, 025602(R) (2003).

[15] G. Assanto, M. Peccianti, K. Brzdakiewicz, A. De Luca, C. Umeton, J. Nonlinear Opt. Phys. Mater. 12, 123 (2003).

[16] M. Peccianti, C. Conti, G. Assanto, J. Nonlin. Opt. Phys. Mater. 12, 525 (2003). 